Max-Planck-Institut für demografische Forschung

Max Planck Institute for Demographic Research

Doberaner Strasse 114 - D-18057 Rostock · GERMANY

Tel +49 (0) 3812081 - 0; Fax +49 (0) 3812081 - 202;

http://www.demogr.mpg.de

MPIDR WORKING PAPER WP 1999-015

NOVEMBER 1999

\title{
How Mortality Improvement Increases Population Growth
}

James W. Vaupel (jwv@demogr.mpg.de)

Vladimir Canudas Romo (canudas@demogr.mpg.de)

C Copyright is held by the authors.

Working papers of the Max Planck Institute for Demographic Research receive only limited review. Views or opinions expressed in working papers are attributable to the authors and do not necessarily reflect those of the Institute. 


\title{
How Mortality Improvement Increases Population Growth
}

\author{
J.W. Vaupel and V. Canudas Romo \\ Max Planck Institute for Demographic Research, Rostock, Germany
}

November 15, 1999

\section{Introduction}

Pseudo-stable and quasi-stable population models were developed by Coale [3], Feichtinger [6], Dinkel [5] and others. Building on an article by Bennett and Horiuchi [2], Preston and Coale [12], Arthur and Vaupel [1], and Kim [10] further extended these models to develop general relationships for arbitrary population surfaces over age and time. In the spirit of these demographers, we present and prove in this article some formulas that capture the impact of mortality improvement on population growth. Goldstein and Schlag [7] also investigate this question but from a different perspective.

It is sometimes forgotten that in addition to babies and immigrants, people whose lives are saved also augment populations. Over the past century, most of the momentous increase in the world's population has been fueled by increased survival. Birth rates have tended to fall, often sharply, but death rates have decreased even faster. As a result, the population of the world has multiplied. In the future, global birth rates may fall below two children per woman, as they have already done in Europe and parts of the Far East. Population declines caused by such low fertility will be somewhat offset by mortality improvements, to the extent such improvements continue and are substantial. Even without net in-migration, a country's "replacement level of fertility" may be less than 2.0 if lifespans continue to lengthen.

Consider a population closed to migration with a continuous population surface $N(x, y)$ over age $x$ and time $y$. See Keiding [9] and Arthur and Vaupel 
[1] for a discussion of this fundamental but elusive quantity, which is often referred to as age-specific population size. Let the intensity of population growth be denoted by

$$
\rho(x, y)=\frac{d N(x, y) / d y}{N(x, y)}=N^{\prime}(x, y)
$$

where the acute accent, here and elsewhere in this article, denotes the relative derivative with respect to time. Let the intensity of population growth for the population as a whole be denoted by

$$
\bar{\rho}(y)=\frac{\int_{0}^{\omega} \rho(x, y) N(x, y) d x}{\int_{0}^{\omega} N(x, y) d x},
$$

where $\omega$ is the highest age attained. Letting

$$
T(y)=\int_{0}^{\omega} N(x, y) d x
$$

be the total population, note that

$$
\bar{\rho}(y)=\dot{T}(y)=\frac{d T(y) / d y}{T(y)} .
$$

The quantity $\bar{\rho}(y)$, which is often called the population growth rate, is of prime interest to us.

Let the intensity of mortality (also known as the hazard of death or force of mortality) be given by

$$
\mu(x, y)=-\frac{d N(x+a, y+a) / d a}{N(x, y)}
$$

and let the intensity of improvement in mortality be denoted by

$$
\ddot{\mu}(x, y)=-\frac{d \mu(x, y) / d y}{\mu(x, y)} .
$$

Our focus is on how $\mu(x, y)$ affects $\bar{\rho}(y)$.

As shown by Preston and Coale [12],

$$
N(x, y)=B(y) s(x, y) R(x, y),
$$


where $B(y)$ denotes the number of births at time $y, s(x, y)$ is the period survival function,

$$
s(x, y)=e^{-\int_{0}^{x} \mu(a, y) d a}
$$

and

$$
R(x, y)=e^{-\int_{0}^{x} \rho(a, y) d a} .
$$

As emphasised by Arthur and Vaupel [1],

$$
N(x, y)=B(y-x) s_{c}(x, y),
$$

where $B(y-x)$ denotes the number of births at time $y-x$ and $s_{c}(x, y)$ is the cohort survival function

$$
s_{c}(x, y)=e^{-\int_{0}^{x} \mu(a, y-x+a) d a} .
$$

It follows from (7) and (10) that

$$
R(x, y)=\frac{B(y-x)}{B(y)} \frac{s_{c}(x, y)}{s(x, y)},
$$

so $R(x, y)$ can be considered to be a cohort-period adjustment that captures the dissimilarity between actual cohort and synthetic period values.

To understand how population growth is related to changes in the number of births, to improvements in mortality, and to the cohort-period adjustment, it makes sense to start with a general result that is useful for a variety of different kinds of decomposition. Consider some total or sum $V(y)$ :

$$
V(y)=\int v(x, y) d x
$$

or

$$
V(y)=\sum_{x} v(x, y)
$$

Suppose

$$
v(x, y)=u_{1}(x, y) u_{2}(x, y) \ldots u_{n}(x, y) .
$$


Then

$$
\dot{V}^{\prime}(y)=\sum_{i=1}^{n} E\left[\dot{u}_{i}(x, y)\right]
$$

where

$$
E\left[\dot{u}_{i}(x, y)\right]=\frac{\int \dot{u}_{i}(x, y) v(x, y) d x}{\int v(x, y) d x}
$$

or

$$
E\left[\dot{u}_{i}(x, y)\right]=\frac{\sum_{x} \dot{u}_{i}(x, y) v(x, y)}{\sum_{x} v(x, y)} .
$$

To prove this result, note that

$$
\dot{v}(x, y)=\dot{u}_{1}(x, y)+\dot{u}_{2}(x, y)+\ldots+\dot{u}_{n}(x, y)
$$

and

$$
\dot{V}^{\prime}(y)=\frac{\int \dot{v}(x, y) v(x, y) d x}{\int v(x, y) d x}
$$

The general result of formula (16) can be used in equation (3) after substituting (7) to decompose population growth into three components:

$$
\bar{\rho}(y)=\dot{B}(y)+\bar{s}(y)+\bar{R}(y)
$$

where $\dot{B}(y)$ is the intensity of change in births, $\bar{s}(y)$ is the average intensity of change in survival,

$$
\bar{s}(y)=\frac{\int_{0}^{\omega} \frac{d s(x, y) / d y}{s(x, y)} N(x, y) d x}{\int_{0}^{\omega} N(x, y) d x}
$$

and $\bar{R}(y)$ is the average intensity of change in the cohort-period adjustment

$$
\bar{R}(y)=\frac{\int_{0}^{\omega} \frac{d R(x, y) / d y}{R(x, y)} N(x, y) d x}{\int_{0}^{\omega} N(x, y) d x} .
$$

It should be noted, however, that the second term on the right-hand side of (21) reflects both current changes in mortality and historical factors that have determined the current population structure. To eliminate the 
influence of past changes in fertility and mortality (and perhaps migration), the following result, due to Vaupel [14], is useful:

$$
E_{w_{2}}(v)-E_{w_{1}}(v)=\frac{\operatorname{Cov}_{w_{1}}(v, \varphi)}{E_{w_{1}}(\varphi)}
$$

where

$$
\varphi \equiv \varphi(x)=\frac{w_{2}(x)}{w_{1}(x)} .
$$

Note that expected values are given by

$$
E_{w_{i}}(v)=\frac{\int_{0}^{\omega} v(x) w_{i}(x) d x}{\int_{0}^{\omega} w_{i}(x) d x}
$$

and the covariance is given by

$$
\operatorname{Cov}_{w_{1}}(v, \varphi)=\frac{\int_{0}^{\omega} v(x) \varphi(x) w_{1}(x) d x}{\int_{0}^{\omega} w_{1}(x) d x}-\frac{\int_{0}^{\omega} v(x) w_{1}(x) d x}{\int_{0}^{\omega} w_{1}(x) d x} \frac{\int_{0}^{\omega} \varphi(x) w_{1}(x) d x}{\int_{0}^{\omega} w_{1}(x) d x}
$$

Formula (24) is readily proved by substitution and simplification.

Results (21) and (24) imply

$$
\bar{\rho}(y)=\dot{B}(y)+\dot{e}_{o}(y)+R^{*}(y)
$$

where

$$
R^{*}(y)=\bar{R}(y)-\operatorname{Cov}_{N}\left(\dot{s}(x, y), R^{-1}\right) \frac{T(y)}{B(y) e_{o}(y)} .
$$

To prove this, let $w_{1}(x)=N(x, y)$ and $w_{2}(x)=B(y) s(x, y)$ and note that

$$
E_{s(x, y)}(\dot{s}(x, y))=\dot{e}_{o}(y)
$$

because

$$
e_{o}(y)=\int_{0}^{\omega} s(x, y) d x
$$

Formula (28) permits decomposition of the current population growth rate into 
(1) the current intensity of change in births,

(2) the current intensity of change in period life expectancy (which captures the impact of current mortality change), and

(3) a residual term that reflects the influence of historical fluctuations that have resulted in a population size and structure that is different from the stationary population size and structure implied by current mortality and births.

Note that (7) implies that

$$
R(x, y)=\frac{N(x, y)}{B(y) s(x, y)}
$$

Regardless of whether a population is open or closed to migration, the value of $R(x, y)$ can thus be interpreted as the ratio of the actual population to the stationary life-table population. Hence, if the actual population structure is the same as the life-table structure, i.e.,

$$
N(x, y)=B(y) s(x, y)
$$

then

$$
R(x, y)=1, \text { for all } \mathrm{x}
$$

and

$$
R^{*}(y)=0 .
$$

Table 1 provides some illustrative examples of the decomposition in (28). The population growth rate was estimated by

$$
\bar{\rho}(y) \approx \frac{\ln \left[\frac{T(y+5)}{T(y-5)}\right]}{10} .
$$

The intensity of change in births was estimated by

$$
\dot{B}(y) \approx \frac{\ln \left[\frac{B(y+5)}{B(y-5)}\right]}{10} .
$$

Similarly, the intensity of change in period life expectancy was estimated by 
Table 1: Decomposition of Population Growth Rate

\begin{tabular}{lccccc}
\hline & Year & $\bar{\rho}(y) \%$ & $\dot{B}(y) \%$ & $\dot{e}_{o}(y) \%$ & $R^{*}(y) \%$ \\
\hline \multirow{3}{*}{ World } & 1960 & 1.92 & 1.40 & 1.21 & -0.69 \\
& 1990 & 1.60 & -0.11 & 0.39 & 1.32 \\
\hline Austria & 2040 & 0.55 & 0.07 & 0.20 & 0.28 \\
Belgium & 1990 & 0.60 & 0.14 & 0.39 & 0.07 \\
Finland & 1990 & 0.27 & 0.13 & 0.30 & -0.16 \\
Germany & 1990 & 0.41 & 0.04 & 0.28 & 0.09 \\
Italy & 1990 & 0.48 & -0.62 & 0.30 & 0.80 \\
United Kingdom & 1990 & 0.12 & -1.02 & 0.30 & 0.84 \\
USA & 1990 & 0.33 & -0.25 & 0.26 & 0.33 \\
\hline Sorre: Data & 1990 & 0.99 & -0.49 & 0.24 & 1.24 \\
\hline
\end{tabular}

Source: Data from Eurostat [4]; World and USA from United Nations [13].

$$
\dot{e}_{o} \approx \frac{\ln \left[\frac{e_{o}(y+5)}{e_{o}(y-5)}\right]}{10} .
$$

Finally, $R^{*}(y)$ was simply estimated as the residual

$$
R^{*}(y)=\bar{\rho}(y)-\dot{B}(y)-\dot{e}_{o}(y) .
$$

In Keyfitz [8] and Vaupel [15] the impact on life expectancy of changes in age-specific death rates is analyzed. Their results, when combined with (28), shed light on how mortality change affects population growth. If the rate of mortality improvement is constant over age,

$$
\mu(x, y)=\dot{\mu}(y), \text { for all } \mathrm{x},
$$

then Keyfitz [8] shows that

$$
\dot{e}_{o}(y)=\dot{\mu}(y) H(y)
$$

where $H(y)$ is given by

$$
H(y)=-\frac{\int_{0}^{\omega} s(x, y) \ln [s(x, y)] d x}{\int_{0}^{\omega} s(x, y) d x}
$$


and can be interpreted as the entropy of the survival function. If mortality follows a Gompertz trajectory,

$$
\mu(x, y)=a_{y} e^{b x}
$$

then Vaupel [15] indicates that

$$
\dot{e}_{o}(y) \approx \frac{\dot{\mu}(y) / b}{e_{o}(y)}
$$

and

$$
e_{o}(y+1)-e_{o}(y) \approx \frac{\dot{\mu}(y+0.5)}{b} .
$$

Note that (45) implies that a constant rate of mortality improvement will continue to increase life expectancy by about the same absolute amount. On the other hand, (44) implies that as life expectancy increases, the relative rate (i.e., intensity) of improvement will fall.

Because Vaupel's derivation of these approximations is in an unpublished working paper (Vaupel [16]), we provide a derivation here. Approximation (45) follows from (44) via the approximation

$$
\dot{e}_{o}(y) \approx \frac{e_{o}(y+1)-e_{o}(y)}{e_{o}(y)} .
$$

Approximation (44) can be derived from (41) by showing that

$$
e_{o}(y) H(y) \approx \frac{1}{b}
$$

Substituting (43) in (8) and then in (42) and then substituting the left hand side of (43), (8) and (31) yields

$$
\begin{gathered}
e_{o}(y) H(y)=\frac{1}{b}\left[\int_{0}^{\omega} \mu(x, y) s(x, y) d x-a_{y} \int_{0}^{\omega} s(x, y) d x\right] \\
=\frac{1}{b}\left[1-a_{y} e_{o}(y)\right] .
\end{gathered}
$$

If $a_{y}<<e_{o}$, as it generally is in low-mortality populations, then the approximation follows. The approximation gets better as mortality improvements are made, because (44) implies that $a_{y}$ declines faster than $e_{o}$ rises. 
Consider now the relationship between the total fertility rate $(T F R)$ and the intensity of change in the number of births. Let $A$ be the average age of childbearing. Then the net reproduction rate $(N R R)$ is approximately given by

$$
N R R \approx \pi s(A) T F R,
$$

where $\pi$ is the proportion of female births and $s(A)$ is a girl's chance of surviving to the average age of childbearing (Coale [3]). Under current conditions in developed countries, $N R R \approx 0.48 T F R$, so that an $N R R$ of about one will be produced by a $T F R$ of about 2.1. In a stable population (i.e., with fixed age-specific fertility and mortality rates), a well-known result from Lotka [11] implies

$$
\dot{B} \approx \frac{N R R-1}{A} .
$$

Assume $R^{*}=0$. Let the $T F R$ be 1.99 , let life expectancy at birth be 80 , and let the pace of mortality improvement be $1.5 \%$ per year (which is close to the average current level in some developed countries). Assume mortality increases exponentially at a rate of 0.1 . Then

$$
\bar{\rho}(y)=\dot{B}(y)+\dot{e}_{o}(y)=-0.0015+0.0019=0.0004 .
$$

Although stylised, this result shows that population growth can be positive even if the $T F R$ is below the so-called replacement level.

\section{References}

[1] Arthur, W.B. and Vaupel, J.W. (1984). "Some general relationships in population dynamics". Population Index, 50(2): 214-26.

[2] Bennett, N. and Horiuchi, S. (1981). "Estimating the completeness of death registration in a closed population". Population Index, 47(2): 207-21. 
[3] Coale, A.J. (1972). The Growth and Structure of Human Populations: A Mathematical Investigation. New York: Princeton University Press.

[4] Eurostat (1998). NewCronos CD 98: Different Statistics from European Countries. Luxemburg.

[5] Dinkel, R.H. (1989). Demographie. Band 1: Bevölkerungsdynamik. Munich: Vahlen.

[6] Feichtinger, G. (1979). Demographische Analyse und populationsdynamische Modelle. Wien New York: Springer-Verlag.

[7] Goldstein and Schlag (forthcoming). "Longer life and population growth". Population and Development Review.

[8] Keyfitz, N. (1977). Applied Mathematical Demography. New York: Wiley.

[9] Keiding, N. (1990). "Statistical inference in the Lexis Diagram". Philosophical Transactions of the Royal Society of London, 487-509.

[10] Kim, Y.J. (1986). "Examination of the generalized age distribution". Demography 23: 451-61.

[11] Lotka, A.J. (1934). Théorie Analytique des associations biologiques. France: Hermann éditeurs.

[12] Preston, S.H. and Coale, A.J. (1982). "Age structure, growth, attrition and accession: a new synthesis". Population Index, 48(2): 217-59.

[13] United Nations Population Division Databases, Population Prospects (1996). United Nations, New York.

[14] Vaupel, J.W. (1992). Analysis of Population Changes and Differences. Unpublished book.

[15] Vaupel, J.W. (1986). "How change in age-specific mortality affects life expectancy". Population Studies 40, 147-157.

[16] Vaupel, J.W. (1985). "How change in age-specific mortality affects life expectancy".WP-85-17. (Laxenburg, Austria: International Institute for Applied Systems Analysis, 1985). 